\title{
Correction to: Inner retinal structure and visual function after idiopathic epiretinal membrane surgery with and without brilliant blue $\mathrm{G}$
}

\author{
Asuka Takeyama $^{1,2} \cdot$ Yutaka Imamura $^{1} \cdot$ Masaki Shibata $^{1} \cdot$ Yuko Komiya $^{1} \cdot$ Masahiro Ishida $^{2,1}$
}

Published online: 13 August 2021

(c) Japanese Ophthalmological Society 2021

Correction to: Japanese Journal of Ophthalmology https://doi.org/10.1007/s10384-021-00851-7

In the Original publication, Table 4 has been published incorrectly, due to error occurred in Copy Editing. The correct version of Table 4 is given in this Correction.

Table 4 The ganglion cell complex thickness to total retinal thickness ratios in the non-dye group and brilliant blue G group

\begin{tabular}{|c|c|c|c|c|c|c|c|c|c|c|}
\hline & \multicolumn{2}{|c|}{1 month } & \multicolumn{2}{|c|}{3 months } & \multicolumn{2}{|c|}{6 months } & \multicolumn{2}{|c|}{12 months } & \multirow[t]{2}{*}{$P$ value } \\
\hline \multicolumn{2}{|c|}{$\mathrm{G} / \mathrm{T}$ ratio $(\%)$} & \multirow{2}{*}{$\begin{array}{l}\text { Mean } \\
28.16\end{array}$} & \multirow{2}{*}{$\frac{S D}{3.56}$} & \multirow{2}{*}{$\frac{\text { Mean }}{26.95}$} & \multirow{2}{*}{$\frac{S D}{3.27}$} & \multirow{2}{*}{$\frac{\text { Mean }}{26.94}$} & \multirow{2}{*}{$\frac{S D}{3.32}$} & \multirow{2}{*}{$\frac{\text { Mean }}{26.85}$} & \multirow{2}{*}{$\frac{S D}{2.87}$} & \\
\hline \multirow[t]{2}{*}{ Average } & Non-dye group & & & & & & & & & \multirow[t]{2}{*}{0.073} \\
\hline & BBG group & 29.45 & 3.04 & 28.25 & 3.12 & 28.05 & 3.15 & 27.74 & 3.10 & \\
\hline \multirow[t]{2}{*}{ Superior } & Non-dye group & 30.00 & 4.17 & 28.99 & 4.03 & 29.29 & 3.73 & 28.83 & 3.89 & \multirow[t]{2}{*}{0.010} \\
\hline & BBG group & 32.08 & 4.74 & 30.76 & 4.47 & 30.47 & 4.84 & 30.79 & 4.41 & \\
\hline \multirow[t]{2}{*}{ Inferior } & Non-dye group & 29.98 & 4.71 & 29.21 & 4.79 & 28.55 & 4.86 & 29.41 & 4.27 & \multirow[t]{2}{*}{0.616} \\
\hline & BBG group & 31.93 & 4.05 & 30.87 & 4.22 & 30.55 & 3.95 & 29.89 & 3.84 & \\
\hline \multirow[t]{2}{*}{ Nasal } & Non-dye group & 27.14 & 4.88 & 25.51 & 3.96 & 25.43 & 4.03 & 25.00 & 3.40 & \multirow[t]{2}{*}{0.163} \\
\hline & BBG group & 28.33 & 4.84 & 26.62 & 4.38 & 26.32 & 4.74 & 25.80 & 4.13 & \\
\hline \multirow[t]{2}{*}{ Temporal } & Non-dye group & 25.52 & 4.07 & 24.10 & 4.13 & 24.50 & 3.78 & 24.19 & 3.76 & \multirow[t]{2}{*}{0.233} \\
\hline & BBG group & 25.48 & 3.30 & 24.76 & 3.98 & 24.86 & 4.01 & 24.46 & 4.45 & \\
\hline
\end{tabular}

The differences in the G/T ratio between groups at 12 months were analyzed using the Mann-Whitney U test

$S D$ standard deviation, $B B G$ Brilliant blue G, $G / T$ ratio ganglion cell complex thickness to total retinal thickness ratio
Original article has been corrected.

The original article can be found online at https://doi.org/10.1007/ s10384-021-00851-7.

Asuka Takeyama asucafejp@yahoo.co.jp

1 Department of Ophthalmology, Teikyo University School of Medicine, University Hospital Mizonokuchi, 5-1-1Takatsu-ku, FutagoKawasaki, Kanagawa 213-8507, Japan

2 Department of Ophthalmology, Toho University Ohashi Medical Center, Tokyo, Japan
Publisher's Note Springer Nature remains neutral with regard to jurisdictional claims in published maps and institutional affiliations. 